\title{
Exact master equations for driven dissipative tight-binding models
}

\author{
Milena Grifoni, ${ }^{1}$ Maura Sassetti, ${ }^{2}$ and Ulrich Weiss ${ }^{3}$ \\ ${ }^{1}$ Institut für Physik, Universität Augsburg, Memminger Straße 6, D-86135 Augsburg, Germany \\ ${ }^{2}$ Istituto di Fisica di Ingegneria, INFM, Università di Genova, Via Dodecaneso 33, I-16146 Genova, Italy \\ ${ }^{3}$ Institut für Theoretische Physik, Universität Stuttgart, Pfaffenwaldring 57, D-70550 Stuttgart, Germany
}

(Received 18 December 1995)

\begin{abstract}
The dynamics of the driven dissipative two-state system is formulated in terms of an exact nonconvolutive master equation. The kernel is expressed as power series in the intersite coupling, in which the lowest order corresponds to the familiar noninteracting-blip approximation. We use this formalism to calculate the kernel systematically in all orders of the intersite coupling for weak damping, and we solve the affiliated master equation for high-frequency driving in analytic form. Our approach finds straightforward generalization to any multistate dissipative tight-binding system.
\end{abstract}

PACS number(s): 05.30. $-\mathrm{d}, 05.40 .+\mathrm{j}, 33.80 . \mathrm{Be}$

The problem of a tight-binding (TB) particle coupled to a thermal bath is of relevance for a great variety of transport processes in physics and chemistry. For the simplest case of two states, a simple and powerful approximation, the noninteracting-blip approximation (NIBA), has been developed by Leggett et al. [1]. For the case of dissipative multistate systems $(N>2)$, the concept of the NIBA has been generalized to the noninteracting-cluster approximation (NICA) [2]. Within this approximation, the dynamics is represented by a single (NIBA), or by a set (NICA) of retarded master equations, in which the kernel or kernel matrix is defined by the sum of those paths which interpolate between two diagonal states of the reduced density matrix without visiting any diagonal state at intermediate times. For $N=2$ (NIBA), the kernel is quadratic in the intersite coupling, while for $N>2$ (NICA), already any even number of hops contributes to the kernel matrix.

An important question is to which degree the dissipative tunneling dynamics is influenced or may be controlled by external time-dependent fields. Recently, the NIBA has been applied to the driven damped two-state system [3-7]. Again one finds that the dynamics is described in terms of retarded master equations, but they are no more in convolutive form. The various limits in which the NIBA becomes asymptotically correct have been discussed for a static bias in Refs. $[1,8]$. For driven systems, the limits were found to be roughly the same [6].

In this Rapid Communication, we show that the exact dynamics of the driven dissipative multistate system can be described by time-retarded nonconvolutive master equations as well. The kernel is represented by a power series in the intersite coupling, and the NIBA corresponds to the lowest order. We discuss a reduction scheme which is possible for high-frequency driving, and we present an approach that allows to compute the dynamics for weak damping systematically in the parameter regime where the NIBA fails. Here we mainly concentrate on the case of two states, and we shortly explain the generalization to any multistate system.

For a qualitative understanding of the environmental effects on the driven TB dynamics, we study the Hamiltonian $H=H_{0}+H_{1}$, where $H_{0}$ represents the two-state system in the standard Pauli spin representation $(\hbar=1)$,

$$
H_{0}=\frac{1}{2}\left\{-\Delta \sigma_{x}+\left[\epsilon_{0}+\hat{\epsilon} f(t)\right] \sigma_{z}\right\} .
$$

Here, $\Delta$ describes the coupling between the two states of distance $a$, and $\epsilon_{0}$ is a static bias energy. Further, we applied an external time-dependent force $\hat{\epsilon} f(t) / a$. The environment is modeled by a term $H_{1}$ describing a harmonic oscillator bath and a bilinear coupling in the coordinates of the bath and of the two-state system.

Suppose that at times $t<t_{0}$ the particle is held at the site $\sigma_{z}=1$ with the bath having a thermal distribution. We then wish to compute the probability $\left\langle\sigma_{z}\left(t, t_{0}\right)\right\rangle \equiv P\left(t, t_{0}\right)$ $=P\left(1, t ; 1, t_{0}\right)-P\left(-1, t ; 1, t_{0}\right)$ at times $t>t_{0}$ for this factorizing initial state. After tracing out the thermal bath $[1,8]$, all environmental effects are captured by the twice-integrated bath correlation function $[1,8]\left(\beta=1 / k_{B} T\right)$

$$
Q(t)=\frac{a^{2}}{\pi} \int_{0}^{\infty} d \omega \frac{J(\omega)}{\omega^{2}} \frac{\cosh [\omega \beta / 2]-\cosh [\omega(\beta / 2-i t)]}{\sinh [\omega \beta / 2]}
$$

where $J(\omega)$ is the spectral density of the heat bath.

Upon summing over the history of the system's visits of the four states of the reduced density matrix, we find the exact formal solution for the evolution of the driven damped system in the form of an expansion in the number of timeordered tunneling transitions. It reads [3]

$$
\begin{aligned}
P\left(t, t_{0}\right)= & +\sum_{n=1}^{\infty}\left(-\Delta^{2}\right)^{n} \int_{t_{0}}^{t} d t_{2 n} \int_{t_{0}}^{t_{2 n}} d t_{2 n-1} \cdots \int_{t_{0}}^{t_{2}} d t_{1} \\
& \times 2^{-n} \sum_{\left\{\xi_{j}= \pm 1\right\}}\left(F_{n}^{(+)} C_{n}^{(+)}+F_{n}^{(-)} C_{n}^{(-)}\right) \\
C_{n}^{(+)}= & \cos \Phi_{n} ; \quad C_{n}^{(-)}=\sin \Phi_{n}
\end{aligned}
$$

Here the $\xi$ charges label the two off-diagonal states of the reduced density matrix, while the sum over the diagonal states visited at intermediate times has already been performed. The phase $\Phi_{n}$ describes the influence of the static and of the time-dependent biasing forces, 


$$
\Phi_{n}=\sum_{j=1}^{n} \xi_{j}\left[\epsilon_{0}\left(t_{2 j}-t_{2 j-1}\right)+g\left(t_{2 j}\right)-g\left(t_{2 j-1}\right)\right],
$$

where $g(t)=\hat{\epsilon} \int^{t} d t^{\prime} f\left(t^{\prime}\right)$.

All the dissipative influences are in the influence functions $F_{n}^{( \pm)}$. Before presenting their forms, we introduce some terminology. The periods $t_{2 j}<t^{\prime}<t_{2 j+1}$, in which the system is in a diagonal state, are usually referred to as sojourns, and the periods $t_{2 j-1}<t^{\prime}<t_{2 j}$, in which the system stays in one of the two off-diagonal states, are called blips (cf. Refs. $[1,8])$. The bath correlations are conveniently expressed in terms of the functions

$$
\begin{gathered}
Q_{j, k}=Q\left(t_{j}-t_{k}\right), \\
\Lambda_{j, k}=Q_{2 j, 2 k}^{\prime}+Q_{2 j-1,2 k}^{\prime}-Q_{2 j, 2 k}^{\prime}-Q_{2 j-1,2 k-1}^{\prime}, \\
X_{j, k}=Q_{2 j, 2 k+1}^{\prime \prime}+Q_{2 j-1,2 k}^{\prime \prime}-Q_{2 j, 2 k}^{\prime \prime}-Q_{2 j-1,2 k+1}^{\prime \prime},
\end{gathered}
$$

where $Q^{\prime}(t)$ and $Q^{\prime \prime}(t)$ are the real and imaginary part of the bath correlation function $Q(t)$ respectively. The function $\Lambda_{j, k}$ describes the interblip correlations of the blip pair $\{j, k\}$, while the function $X_{j, k}$ describes the correlations of the blip $j$ with a preceding sojourn $k$. For $k=0$, we have $X_{j, 0}=Q_{2 j, 1}^{\prime \prime}-Q_{2 j-1,1}^{\prime \prime}$.

Then, all intrablip and interblip correlations of $n$ blips are combined in the expression

$$
G_{n}=\exp \left(-\sum_{j=1}^{n} Q_{2 j, 2 j-1}^{\prime}-\sum_{j=2}^{n} \sum_{k=1}^{j-1} \xi_{j} \xi_{k} \Lambda_{j, k}\right) .
$$

Upon introducing the influence phases describing the correlations between the $k$ th sojourn and the $n-k$ succeeding blips, $\eta_{n, k}=\sum_{j=k+1}^{n} \xi_{j} X_{j, k}$, the full influence functions take the compact form

$$
F_{n}^{(+)}=G_{n} \prod_{k=0}^{n-1} \cos \eta_{n, k} ; \quad F_{n}^{(-)}=F_{n}^{(+)} \tan \eta_{n, 0} .
$$

In the exact formal solution (2), all the flip times $\left\{t_{j}\right\}$ are correlated with each other by the bath correlation functions (5) encapsulated in the influence functions $F_{n}^{( \pm)}$. In addition, they are also influenced by the actions $g\left(t_{j}\right)$ of the external field which are regulating the bias phases in $\Phi_{n}$. Notwithstanding these formidable intricacies, the full dynamics of the conditional probabilities $P\left(i, t ; j, t_{0}\right)$ can be described by a set of exact generalized master equations (GME's)

$$
\dot{P}\left(i, t ; j, t_{0}\right)=\sum_{k} \int_{t_{0}}^{t} d t^{\prime} K\left(i, t ; k, t^{\prime}\right) P\left(k, t^{\prime} ; j, t_{0}\right) .
$$

The kernels fulfill the sum rule $\sum_{i} K\left(i, t ; j, t^{\prime}\right)=0$, which provides conservation of probability. Upon introducing $K^{( \pm)}=K(1,1) \pm K(-1,-1)$, and using $P(1,1)+P(-1,1)$ $=1$, Eq. (7) can be rewritten as a GME for $P\left(t, t_{0}\right)$,

$$
\dot{P}\left(t, t_{0}\right)=\int_{t_{0}}^{t} d t^{\prime}\left[K^{(+)}\left(t, t^{\prime}\right) P\left(t^{\prime}, t_{0}\right)+K^{(-)}\left(t, t^{\prime}\right)\right] .
$$

To proceed from (2) to (8), we define modified influence functions $\tilde{F}_{n}^{( \pm)}$involving suitable subtractions with products of lower order influence functions,

$$
\begin{aligned}
\tilde{F}_{n}^{( \pm)}= & F_{n}^{( \pm)}-\sum_{j=2}^{n}(-1)^{j} \sum_{m_{1}, \cdots, m_{j}} F_{m_{1}}^{(+)} F_{m_{2}}^{(+)} \cdots F_{m_{j}}^{( \pm)} \\
& \times \delta_{m_{1}}+\cdots+m_{j}, n
\end{aligned}
$$

Here the inner sum is over positive integers $m_{j}$. For instance, the $n=3$ term reads

$$
\tilde{F}_{3}^{( \pm)}=F_{3}^{( \pm)}-F_{2}^{(+)} F_{1}^{( \pm)}-F_{1}^{(+)} F_{2}^{( \pm)}+F_{1}^{(+)} F_{1}^{(+)} F_{1}^{( \pm)} .
$$

By definition, each subtraction involves again time ordering for the $2 n$ flip times with $t_{1}$ being the rightmost. In the subtracted terms, the bath correlations are only inside of each of the individual factors $F_{m_{j}}^{( \pm)}$, and there are no correlations between these factors.

With the modified influence functions $\tilde{F}_{n}^{( \pm)}$, the kernel $K^{( \pm)}\left(t, t^{\prime}\right)$ of the GME is defined by the series expression

$$
\begin{aligned}
K^{( \pm)}\left(t, t^{\prime}\right)= & \sum_{n=1}^{\infty}\left(-\Delta^{2}\right)^{n} \int_{t^{\prime}}^{t} d t_{2 n-1} \cdots \int_{t^{\prime}}^{t_{3}} d t_{2} \\
& \times 2^{-n} \sum_{\left\{\xi_{j}= \pm 1\right\}} \tilde{F}_{n}^{( \pm)} C_{n}^{( \pm)} .
\end{aligned}
$$

The product function $\tilde{F}_{n}^{( \pm)} C_{n}^{( \pm)}$depends on $2 n$ flip times, and the first and the last one are identified with $t^{\prime}$ and $t$, respectively. All intermediate flip times are integrated over in the expressions for the kernels $K^{( \pm)}\left(t, t^{\prime}\right)$.

Upon comparing the iterative solution of (8) and (9) with the time derivative of (2), it is straightforward to see that they are identical. Thus the GME (8) with the kernels (9) describes the exact dynamics of the damped, externally driven TB particle.

In practical calculations, it is suggestive to truncate the series (9) at a given order, and then solve the GME numerically without further approximation. For instance, if we neglect all interblip correlations $\left(\Lambda_{j, k}=0\right)$ and all blip-sojourn correlations $\left(X_{j, k}=0\right.$ for $\left.j \neq k+1\right)$, the influence functions $\tilde{F}_{n}^{( \pm)}$are zero for all $n>1$, and the kernels reduce to the lowest order expressions

$$
\begin{aligned}
& K_{1}^{(+)}\left(t, t^{\prime}\right)=-\Delta^{2} e^{-Q^{\prime}\left(t-t^{\prime}\right)} \cos \left[Q^{\prime \prime}\left(t-t^{\prime}\right)\right] C_{1}^{(+)}\left(t, t^{\prime}\right), \\
& K_{1}^{(-)}\left(t, t^{\prime}\right)=-\Delta^{2} e^{-Q^{\prime}\left(t-t^{\prime}\right)} \sin \left[Q^{\prime \prime}\left(t-t^{\prime}\right)\right] C_{1}^{(-)}\left(t, t^{\prime}\right) .
\end{aligned}
$$

It is clear from these prescriptions that the resulting GME fully corresponds to the familiar noninteracting-blip approximation [1]. Now, by taking into account subsequent terms of the expansion (9), corrections to the NIBA may be calculated systematically.

In the evaluation of (8) with (9), one encounters two major difficulties which have different origin.

The first difficulty is due to the nonconvolutive form of the bias phase $\Phi_{n}$. For periodic driving with fundamental frequency $\Omega$, the property $g(t)=g(t+2 \pi / \Omega)$ suggests to expand $K^{( \pm)}\left(t, t^{\prime}\right)$ and $P\left(t, t^{\prime}\right)$ into Fourier series, 


$$
\begin{gathered}
P\left(t, t^{\prime}\right)=\sum_{m} p_{m}\left(t-t^{\prime}\right) e^{-i m \Omega t^{\prime}}, \\
K^{( \pm)}\left(t, t^{\prime}\right)=\sum_{m} k_{m}^{( \pm)}\left(t-t^{\prime}\right) e^{-i m \Omega t^{\prime}} .
\end{gathered}
$$

Then we obtain coupled integro-differential equations of convolutive type for the Fourier functions $p_{n}(\tau)$,

$$
\begin{aligned}
\dot{p}_{n}(\tau)= & \int_{0}^{\tau} d \tau^{\prime}\left[\sum_{m} k_{m}^{(+)}\left(\tau-\tau^{\prime}\right) p_{n-m}\left(\tau^{\prime}\right) e^{-i m \Omega \tau^{\prime}}\right. \\
& \left.+k_{n}^{(-)}\left(\tau-\tau^{\prime}\right) e^{-i n \Omega \tau^{\prime}}\right]
\end{aligned}
$$

which may be solved by Laplace transformation and subsequent use of recursion relations [6].

The second remaining difficulty originates from the bath correlations. These make the analytical calculation of higher order terms in the series (9) extremely troublesome. Even the numerical computation of the kernels is not so straightforward because of the destructive interference of the numerous contributions.

So far, apart from the discussion of the NIBA, our treatment has been exact. Moreover, the form (7) of the GME is exact for any $N$-state TB system, if we identify $P\left(i, t ; j, t^{\prime}\right)$ and $K\left(i, t ; j, t^{\prime}\right)$ with the complete set of $N \times N$ conditional probabilities and kernels, respectively. If the kernels are restricted to the subsets of irreducible neutral $\xi$-charge clusters, Eq. (7) describes the NICA.

In the remainder, we restrict the attention to the two-state system, and we consider the limit of nonadiabatic driving and weak damping.

When the characteristic memory time $\tau_{K}$ of the kernels is of the order of $2 \pi / \Omega$ or smaller, many terms $p_{n}\left(t-t^{\prime}\right)$ contribute to $P\left(t, t^{\prime}\right)$. With increasing frequency $\Omega$, the period $2 \pi / \Omega$ eventually gets small compared to $\tau_{K}$, and the set of equations (10) becomes restricted to the lowest Fourier components. For high frequency, the essential dynamics of $P\left(t, t^{\prime}\right)$ is described by $p_{0}\left(t-t^{\prime}\right)$. The reduction to the $n=0$ term is equivalent to taking the average of the phase factor of the driving field for fixed length $t-t^{\prime}$. Then $\left\langle K^{( \pm)}\left(t, t^{\prime}\right)\right\rangle=k_{0}^{( \pm)}\left(t-t^{\prime}\right)$, and Eq. (10) reduces to a single convolutive master equation,

$$
\dot{p}_{0}(\tau)=\int_{0}^{\tau} d \tau^{\prime}\left[k_{0}^{(+)}\left(\tau-\tau^{\prime}\right) p_{0}\left(\tau^{\prime}\right)+k_{0}^{(-)}\left(\tau-\tau^{\prime}\right)\right]
$$

In this limit, the steady state reached at long times does not show periodic oscillations, since it is represented by the $n=0$ Fourier term only. The characteristic memory times of the kernels depend on a subtle interplay of the various inverse time scales $\Delta, \epsilon_{0}, \hat{\epsilon}$, and of the intrablip and interblip correlations. While the former are temperature independent, the bath correlations depend strongly on temperature. Typically, the range of the bath correlations is increased as temperature is decreased. It is intuitively clear that, for sufficiently high frequency, we may perform the average of the phase factor of the driving field separately for each indi- vidual blip contributing to (9). Upon taking the average for fixed blip length $\tau_{j}=t_{2 j}-t_{2 j-1}$, we have

$$
\begin{aligned}
& \left\langle C_{1}^{(+)}\left(t_{2 j}, t_{2 j-1}\right)\right\rangle \equiv c_{1}^{(+)}\left(\tau_{j}\right)=\cos \left(\epsilon_{0} \tau_{j}\right) b_{0}\left(\tau_{j}\right), \\
& \left\langle C_{1}^{(-)}\left(t_{2 j}, t_{2 j-1}\right)\right\rangle \equiv c_{1}^{(-)}\left(\tau_{j}\right)=\sin \left(\epsilon_{0} \tau_{j}\right) b_{0}\left(\tau_{j}\right) .
\end{aligned}
$$

For harmonic driving, $f(t)=\cos (\Omega t)$, the function $b_{0}(\tau)$ takes the form $b_{0}(\tau)=J_{0}((2 \hat{\epsilon} / \Omega) \sin (\Omega \tau / 2))$, where $J_{0}(z)$ is a Bessel function of the first kind.

For weak damping, nonzero bias, and low temperatures, the noninteracting-blip assumption breaks down, since the bath correlations $\Lambda_{j, k}$ and $X_{j, k}$ contribute to effects in linear order of $J(\omega)$. We have been able to sum the full series (9) in the weak-damping limit. To linear order in the bath correlations, we find following Ref. [9] (we drop the $n=0$ Fourier index henceforth)

$$
\begin{aligned}
k^{(+)}(\tau)= & -\Delta^{2} c_{1}^{(+)}(\tau)\left[1-Q^{\prime}(\tau)\right]-\int_{0}^{\tau} d t_{3} \int_{0}^{t_{3}} d t_{2} \\
& \times \Delta^{4} c_{1}^{(-)}\left(\tau-t_{3}\right) p^{(0)}\left(t_{3}-t_{2}\right) c_{1}^{(-)}\left(t_{2}\right) \\
& \times\left[Q^{\prime}(\tau)+Q^{\prime}\left(t_{3}-t_{2}\right)-Q^{\prime}\left(t_{3}\right)-Q^{\prime}\left(\tau-t_{2}\right)\right] \\
k^{(-)}(\tau)= & -\Delta^{2} c_{1}^{(-)}(\tau) Q^{\prime \prime}(\tau)-\int_{0}^{\tau} d t_{3} \int_{0}^{t_{3}} d t_{2} \\
& \times \Delta^{4} c_{1}^{(-)}\left(\tau-t_{3}\right) p^{(0)}\left(t_{3}-t_{2}\right) c_{1}^{(+)}\left(t_{2}\right) \\
& \times\left[Q^{\prime \prime}(\tau)-Q^{\prime \prime}\left(t_{3}\right)\right] .
\end{aligned}
$$

In these exact weak-coupling expressions, the respective first term represents the NIBA. The residual term has been written in the form of a double-blip contribution dressed at the interblip interval $t_{3}-t_{2}$ with the term $p^{(0)}\left(t_{3}-t_{2}\right)$ which takes into account all the tunneling transitions of the undamped system in this time regime. In the weak-damping limit, the flip times in the second terms of $k^{( \pm)}(\tau)$ in (11) are free of bath correlations except the ones belonging to the first and to the last blip.

It is convenient to solve the GME for $p(t)$ by Laplace transformation. The Laplace transform of $p(t)$ is represented by a set of simple poles, if the Taylor expansion of the Bessel function in $b_{0}(\tau)$ is truncated at a given order (our notation covers this general case). For $\hat{\epsilon} / \Omega \ll 1$, it is sufficient to retain the terms of order $(\hat{\epsilon} / \Omega)^{2}$. In this approximation, the undamped driven system is characterized by three bias frequencies, $\epsilon_{1}=\epsilon_{0}, \epsilon_{2}=\epsilon_{0}+\Omega, \epsilon_{3}=\epsilon_{0}-\Omega$, and by three tunneling frequencies $\nu_{1}, \nu_{2}$, and $\nu_{3}$. The squares of these frequencies are the solutions of a cubic equation for $\nu^{2}$, $\nu^{6}-a_{4} \nu^{4}+a_{2} \nu^{2}-a_{0}=0$. The coefficients are

$$
\begin{gathered}
a_{0}=\epsilon_{1}^{2} \epsilon_{2}^{2} \epsilon_{3}^{2}+\left[\Delta_{1}^{2} \epsilon_{2}^{2} \epsilon_{3}^{2}+\text { cycl. }\right], \\
a_{2}=\Delta_{1}^{2}\left(\epsilon_{2}^{2}+\epsilon_{3}^{2}\right)+\epsilon_{2}^{2} \epsilon_{3}^{2}+\text { cycl. } \\
a_{4}=\epsilon_{1}^{2}+\Delta_{1}^{2}+\text { cycl. }
\end{gathered}
$$

with the modified tunneling matrix elements

$$
\Delta_{1}^{2}=\left(1-\hat{\boldsymbol{\epsilon}}^{2} / 2 \Omega^{2}\right) \Delta^{2} ; \quad \Delta_{2}^{2}=\Delta_{3}^{2}=\left(\hat{\epsilon}^{2} / 2 \Omega^{2}\right) \Delta^{2} .
$$


The undamped system shows coherent oscillations,

$$
p^{(0)}(t)=p_{0}+\sum_{j} p_{j} \cos \left(\nu_{j} t\right)
$$

with the amplitudes $\left(p_{1}, p_{2}, p_{3}\right.$ cycl.)

$$
p_{1}=\frac{\left(\nu_{1}^{2}-\epsilon_{1}^{2}\right)\left(\nu_{1}^{2}-\epsilon_{2}^{2}\right)\left(\nu_{1}^{2}-\epsilon_{3}^{2}\right)}{\nu_{1}^{2}\left(\nu_{1}^{2}-\nu_{2}^{2}\right)\left(\nu_{1}^{2}-\nu_{3}^{2}\right)} ; \quad p_{0}=\prod_{j=1}^{3} \frac{\epsilon_{j}^{2}}{\nu_{j}^{2}},
$$

obeying the sum rule $\Sigma_{j=0}^{3} p_{j}=1$.

Upon inserting (12) and the spectral representation for $Q(\tau)$, and interchanging the frequency integral with the time integrals, the kernels (11) can be calculated in explicit form. As a result of the bath influence, the poles of the Laplace transform of $p^{(0)}(t)$ are shifted from the imaginary axis into the negative-real halfplane, and there appears an additional pole at the origin. The residuum of this pole (denoted by $p_{\infty}$ ) represents the equilibrium population reached at long times. The dynamics is described by the expression [10]

$$
p(t)=\sum_{j=1}^{3} p_{j} \cos \left(\nu_{j} t\right) e^{-\gamma_{j} t}+\left(p_{0}-p_{\infty}\right) e^{-\gamma_{0} t}+p_{\infty}
$$

with the damping rates and equilibrium distribution

$$
\begin{gathered}
\gamma_{0}=\frac{\pi}{2} \sum_{i=1}^{3} A_{i}^{2} S\left(\nu_{i}\right), \\
\gamma_{j}=\frac{\pi}{4}\left\{A_{j}^{2} S\left(\nu_{j}\right)+C_{j, j}^{2} S(0)+\frac{1}{2} \sum_{i \neq j}\left[C_{j,-i}^{2} S\left(\nu_{j}+\nu_{i}\right)\right.\right. \\
\left.\left.+C_{j, i}^{2} S\left(\left|\nu_{j}-\nu_{i}\right|\right)\right]\right\}, \\
p_{\infty}=-\frac{a^{2}}{2 \gamma_{0}} \sum_{j=1}^{3} A_{j} B_{j} J\left(\nu_{j}\right) .
\end{gathered}
$$

The function $S(\omega)$ depends linearly on the spectral density $J(\omega)$ and on the familiar function $\operatorname{coth}(\beta \omega / 2)$ which describes emission and absorption of a single boson with frequency $\omega$ at thermal equilibrium,

$$
S(\omega)=\left(a^{2} / \pi\right) J(\omega) \operatorname{coth}(\beta \omega / 2) .
$$

The amplitudes $A_{i}, B_{i}, C_{i, j}$ are weighting the various singleboson processes which contribute to the rate expressions, and they are given by

$$
\begin{gathered}
A_{i}=\sqrt{p_{0} p_{i}} \sum_{k=1}^{3} \frac{\nu_{i} \Delta_{k}^{2}}{\epsilon_{k}\left(\nu_{i}^{2}-\epsilon_{k}^{2}\right)} ; \quad B_{i}=\sqrt{p_{0} p_{i}} \sum_{k=1}^{3} \frac{\Delta_{k}^{2}}{\nu_{i}^{2}-\epsilon_{k}^{2}}, \\
C_{i, \pm j}=\sqrt{p_{i} p_{j}} \sum_{k=1}^{3} \frac{\left(\nu_{i} \pm \nu_{j}\right) \epsilon_{k} \Delta_{k}^{2}}{\left(\nu_{i}^{2}-\epsilon_{k}^{2}\right)\left(\nu_{j}^{2}-\epsilon_{k}^{2}\right)} .
\end{gathered}
$$

These expressions describe the dynamics in the weakcoupling limit for any non-sub-Ohmic spectral density. In the super-Ohmic case, $\lim _{\omega \rightarrow 0} J(\omega) / \omega \rightarrow 0$, the term $S(0)$ is zero, while for Ohmic damping $S(0) \propto T$. In the sub-Ohmic case, $S(0)$ diverges. This indicates that sub-Ohmic damping is so effective that there is no consistent weak-coupling limit of the form discussed here.

Within the NIBA, the relaxation rate $\gamma_{0}$ takes the form $\gamma_{0}=(\pi / 2) p_{0} \Sigma_{i}\left(\Delta_{i}^{2} / \epsilon_{i}^{2}\right) S\left(\epsilon_{i}\right)$, while the damping rates $\gamma_{j}$ $(j=1,2,3)$ are linear combinations of $S\left(\nu_{j} \pm \epsilon_{i}\right)$ where $i=1,2,3$. Also, $p_{\infty}$ is expressed in terms of $\epsilon_{j}$, instead of the proper frequencies $\nu_{j}$. It is now clear that the NIBA does not correctly take into account the energy shifts induced by tunneling and the amplitudes for the various single-phonon processes. We have to return to the more advanced treatment given here whenever the energy shifts induced by tunneling are essential.

This work was supported in part by the Deutsche Forschungsgemeinschaft (DFG).
[1] A.J. Leggett, S. Chakravarty, A.T. Dorsey, M.P.A. Fisher, A. Garg, and W. Zwerger, Rev. Mod. Phys. 59, 1 (1987).

[2] R. Egger, C.H. Mak, and U. Weiss, Phys. Rev. E 50, R655 (1994).

[3] M. Grifoni, M. Sassetti, J. Stockburger, and U. Weiss, Phys. Rev. E 48, 3497 (1993).

[4] Yu. Dakhnovskii, Phys. Rev. B 49, 4649 (1994); Ann. Phys. 230, 145 (1994).

[5] I.A. Goychuk, E.G. Petrov, and V. May, Phys. Rev. E 52, 2392 (1995).

[6] M. Grifoni, M. Sassetti, P. Hänggi, and U. Weiss, Phys. Rev. E 52, 3596 (1995).

[7] D.G. Evans, R.D. Coalson, H.J. Kim, and Yu. Dakhnovskii,
Phys. Rev. Lett. 75, 3649 (1995); Yu. Dakhnovskii and R.D. Coalson, J. Chem. Phys. 103, 2908 (1995).

[8] U. Weiss, Quantum Dissipative Systems, Series in Modern Condensed Matter Physics, Vol. 2 (World Scientific, Singapore, 1993).

[9] U. Weiss and M. Wollensak, Phys. Rev. Lett. 62, 1663 (1989); R. Görlich, M. Sassetti, and U. Weiss, Europhys. Lett. 10, 507 (1989).

[10] We have disregarded real-valued shifts of the oscillation frequencies $\nu_{j}$, and shifts of the amplitude factors $p_{j}(j=0, \ldots, 3)$ caused by the environmental coupling, as they are of secondary interest here. 